\title{
Analysis of the associations between variables derived throughout velocity-based training device and jumping performances in youth soccer players: Multiple regression study
}

\author{
Vlade Bendic ${ }^{1}$, Barbara Gilic ${ }^{1,2}$, Damir Lastre ${ }^{3}$, Ivan Peric ${ }^{4}$, and Damir Sekulic ${ }^{1 凶}$ \\ ${ }^{1}$ Faculty of Kinesiology, University of Split, Split, Croatia; ${ }^{2}$ Faculty of Kinesiology, University of Zagreb, Zagreb, Croatia; ${ }^{3}$ Fitness Center \\ Joker, Split, Croatia; and ${ }^{4}$ Faculty of Dental Medicine and Health, Josip Juraj Strossmayer University of Osijek, Osijek, Croatia
}

\begin{abstract}
Background: It has been suggested that velocity-based training (VBT) improves real-life performance in sports, such as jumping, but studies rarely examined the associations between variables derived during VBT (VBT-variables) and jumping performances. Objective: The aim of this study was to investigate the associations between VBT-variables derived during deadlift exercise executed at different loads and anthropometric variables, and vertical and horizontal jump performance in young athletes. Methods: Seventeen youth soccer players (16-18 years old) were included. Predictors were body height, body mass, body fat percentage, and VBT-variables (force, power, and velocity) measured at different loads (45\%, 55\%, 65\%, 75\%, 85\%, and 95\%) of a one-repetition maximum deadlift (1RM). Criteria included tests of vertical and horizontal jumping performance. Results: The VBT-variables are more strongly correlated with horizontal jumps (Pearson's $r$ up to .81) than vertical jumps (Pearson's $r$ up to .75). The VBT-variables for the deadlift recorded at lower loads stronger correlated with jumping performance than the VBT-variables recorded at higher loads. Conclusions: Results of the study suggest that light-to-moderate loads ( $45 \%-65 \%$ of $1 \mathrm{RM})$ maximize power output and are therefore more strongly related to jumping performance in youth athletes. Further intervention studies aimed at the improvement of jumping performances using the VBT are warranted.
\end{abstract}

Keywords: youth sports, football, physical exertion, resistance training, strength, conditioning

\section{Introduction}

Training with external loads, known as resistance training, is an efficient method for improving sports performance, as it promotes muscle hypertrophy and increases the maximal power, rate of force development, and power output (Schoenfeld et al., 2016). Although there are numerous methods for prescribing training loads, the most common method is traditionally called percentage-based training (PBT). PBT prescribes relatively submaximal loads on the basis of the previously estimated one-repetition maximum (1RM; Helms et al., 2018). However, the PBT method becomes relatively problematic when daily fluctuations in strength and power are considered. Specifically, daily fluctuations in strength have been shown to be $18 \%$ above or below the previously estimated 1RM (Banyard et al., 2019). Therefore, training based on the velocity of moving the load (velocity-based training - VBT) is becoming increasingly popular as a training method.

The VBT method involves the use of wearable accelerometers (e.g., PUSH band) and linear position transducers (e.g., GymAware) designed for measuring the velocity of the movement during exercise (e.g., barbell velocity during deadlift; Banyard et al., 2019). Specifically, using linear position transducers and accelerometers, the load velocity is calculated, and an individual load-velocity profile is created. As a result, the coach can precisely prescribe and adjust the load based on the velocity during the training (van den Tillaar \& Ball, 2019). However, it must be highlighted that athletes have to perform the exercise with the maximal intended movement velocity. Otherwise, the velocity of the barbell will not be reliable, and the prescribed load would be underestimated (Mann et al., 2015). Finally, VBT provides immediate feedback about the execution of the exercise/movement, which allows objective modifications of the load, identifies optimal loads and velocities for training to improve the specificity of training, and reduces fatigue after exercise (Banyard et al., 2019; Hughes et al., 2019; Mann et al., 2015). Thus, VBT is used in different exercises/movements depending on the sport requirements and is used for enhancing specific sports performances (Mann et al., 2015).

Studies have investigated the effects of VBT on performance in certain sports. Dorrell et al. (2020) noted that six weeks of a VBT intervention led to increases in maximal strength in the back squat, bench press, overhead

$\triangle$ Corresponding author: Damir Sekulic, e-mail dado@kifst.hr, ORCID ${ }^{\circledR}$ record https://orcid.org/0000-0001-8022-7886

Article history: Received January 25 2021, Accepted September 14 2021, Published October 42021

Copyright: (๑ 2021 The Author(s). Published by Palacký University Olomouc. This is an open access article distributed under the terms of the Creative Commons Attribution License (https://creativecommons.org/licenses/by/4.0/), which permits unrestricted use, distribution, and reproduction in any medium, provided the original author and source are credited. This license does not cover any third-party material that may appear with permission in the article. 
press, and deadlift and increased jumping height among experienced trained men. Randell et al. (2011) reported that professional rugby players displayed improvements in the vertical jump, the horizontal jump, and sprints at 10 , 20 , and 30 meters as a result of 6 weeks of squat jump exercises performed in accordance with the VBT method with instantaneous feedback. Furthermore, Dolezal et al. (2016) reported that the vertical jump height and maximal strength in squats improved in track and field athletes after 12 weeks of a VBT intervention.

Several studies have compared the effects of VBT and PBT methods. Specifically, Banyard et al. (2019) reported that compared with PBT, VBT enables individuals to move at higher velocities, prevents unnecessary additional mechanical stress, and yields similar power outputs. Similarly, Orange et al. (2020) compared the effects of VBT and PBT on jump, speed, and strength performance among academy rugby league players and noted larger improvements in maximal strength in the back squat and vertical jump height and lower perceptual training stress in the VBT group. Collectively, previous research has indicated that VBT is applicable for and effective in enhancing sports performance, with an emphasis on improvements in strength and power, with reduced training load.

Generally, it is believed that VBT improves real-life performance in sports (e.g., jumping, throwing, sprinting; Orange et al., 2020). Therefore, correlations between VBTvariables and performance in explosive movements (such as jumps) should exist. Indeed, some research has shown that resistance training with high movement velocities and lightto-moderate loads (30\%-60\% of 1RM) leads to improvements in vertical jump performance (González-Badillo et al., 2015; Ortega et al., 2020). Accordingly, correlations between VBT-variables at a certain percentage of 1RM and jumping performance can be expected. However, whether correlations exist between variables associated with VBT at different $1 \mathrm{RM}$ percentages and different types of jumps is unknown. This information is particularly important, given that different types of jumps should not necessarily be considered together in trained subjects and are influenced by various factors (Pehar et al., 2017).

Horizontal and vertical jumps differ in joint motions and muscle activation. Precisely, the hip joint motion is greater in the horizontal jump compared with the vertical jump (Nagano et al., 2007). In vertical jump, the trunk must be near straight; thus, smaller action of the hip joint is required. On the other hand, in the horizontal jump, the whole body has to be tilted forward which requires increased hip joint motion. Therefore, hip joint extensor muscles are more activated (gluteal muscles and hamstrings) during the horizontal jump than in the vertical jump (Nagano et al., 2007). As a result, it is reasonable to expect that variables recorded while performing a deadlift with different percentages of 1RM are associated with horizontal and vertical jump performance to different extents, especially when different variables of VBT (i.e., velocity, force, power) and different exercise loads are considered. This was recently partly supported by preliminary research (Bendic et al., 2021).
It is well known that different loads produce different kinetic variables. Precisely, the inverse relationship between load and velocity is commonly accepted; that is, velocity decreases with increasing loads (Picerno et al., 2016). Moreover, maximal force is produced at maximal loads; therefore, velocity is also lowering with increasing force. Power is a product of force and velocity and can be described as a parabolic-shaped curve when expressed as a function of velocity. This means that the highest power is obtained at moderate velocities, which correspond to moderate loads (Picerno et al., 2016). VBT requires and elicits maximal movement velocities. Therefore, it is of particular interest to establish associations between different VBT-variables and movements that represent high amounts of velocity and power (i.e., jumps). Supportively, it was established that deadlift performance at maximal intended velocity increased the peak force, velocity, and power variables compared to lifts with submaximal velocities (Swinton et al., 2011).

The main aim of this research was to determine the associations between variables of VBT for the deadlift exercise performed with the maximal intended velocity with different loads and jumping performances in young athletes. An additional aim was to determine the differences within each VBT-variable evidenced at various loads during deadlift exercise. Specifically, we examined horizontal and vertical jumps, and deadlift performance was chosen because of its similarity with jumping performance (Malyszek et al., 2017). We hypothesized that VBT-variables derived at lower percentages of 1RM could predict jumping performance better than VBT-variables derived at higher loads.

\section{Methods}

\section{Subjects}

Seventeen soccer players (16-18 years of age) volunteered to participate in the study (four central defenders, three fullbacks, four central midfielders, three wingers and three forwards). They played junior competitions at the national level of their age. All subjects had practiced soccer for at least eight years, had at least two years of experience in resistance training and had been continuously involved in resistance training for more than six months before the investigation. They did not have any injuries or illnesses that could have impaired their performance at the time of the investigation. The study was approved by the Ethical Board of the University of Split, Faculty of Kinesiology, Split, Croatia. The subjects were informed about the purpose, aims, and procedures of the investigation before the start of the investigation and signed informed consent forms. Parents or legal guardians signed informed consent for participants younger than 18 years of age.

\section{Variables and testing procedures}

Apart from anthropometric/body composition indices, this study included the following variables: 1RM measured with the wearable accelerometer used for the VBT method and parameters assessed at different percentages of 1RM 
(VBT-variables), vertical jump performance (measured by the Sargent jump - SJ), horizontal jump performance (measured by the standing broad jump - BJ). VBT-variables included average (AV) and peak velocity (PV), relative average (AF) and peak force (PF), and relative average (AP) and peak power (PP).

The VBT-variables were measured by the PUSH Band 2.0 wearable accelerometer (PUSH, Toronto, ON, Canada). The PUSH Band 2.0 was attached to the middle of the front side of the hexagonal barbell. Execution of the deadlift exercise and positioning of the PUSH Band 2.0 is shown in Figure 1. The PUSH device consists of a gyroscope and a 3-axis accelerometer. PUSH Band 2.0 detects the beginning and the end of the concentric part of the exercise with proprietary algorithms. The sampling rate is $200 \mathrm{~Hz}$. Calculation of VBT-variables is explained in detail elsewhere (Balsalobre-Fernández et al., 2016). The PUSH Band 2.0 was linked to iOS device (PUSH app v. 7.19.0). The load was selected in the PUSH app for each set. PUSH Band does not require calibration (Balsalobre-Fernández et al., 2016). The loads during the assessment of VBTvariables were determined according to previously assessed 1RM. The VBT-variables were recorded at loads ranging from $45 \%$ to $95 \%$ of pre-determined 1RM, as suggested by Jovanović and Flanagan (2014). VBT-variables have not been measured for loads under $45 \%$ of $1 \mathrm{RM}$ as PUSH Band displayed as not reliable at lighter loads (Chéry \& Ruf, 2019). We exported the VBT-variables from the PUSH app and used the data for later calculations. The reliability of the device in free-weight exercise was confirmed and presented in detail elsewhere (van den Tillaar \& Ball, 2019).

The SJ was measured by standardized equipment (ELAN, Begunje, Slovenia) and was used to assess vertical jumping performance. The participants stood laterally to the wall with their feet shoulder-width apart, and the maximal reach of one arm was recorded. The participants were instructed to perform a vertical jump with countermovement and at the point of maximal reach, leave the mark with chalk on the dark wall. The jump result was displayed in $\mathrm{cm}$ and calculated by subtracting the standing reach value from the maximal jumping reach value. The participants performed three jumping trials (with 30 seconds of rest between trials), and the highest jump was included in further analyses. The SJ has previously been shown to be valid for assessing explosive power among youth soccer players (de Salles et al., 2012).

The BJ was used to assess horizontal jumping performance. The participants stood behind a starting line that was marked on the ground and were instructed to jump as far as possible by using the arm swing. The distance from the starting line to the last recorded trace of the jump represented the result of the test (in $\mathrm{cm}$ ). The players performed three trials with 30 seconds of rest between the trials. A standardized measuring mat was used (ELAN, Begunje, Slovenia), and the longest jump was included in further analyses.

The anthropometric variables were measured by experienced investigators using standardized equipment. The anthropometric variables included body height $(\mathrm{BH})$, body mass $(\mathrm{BM})$, the percentage of body fat $(\% \mathrm{BF})$, measured with a measuring scale (model MC780MA, TANITA, Tokyo, Japan).

\section{Testing protocol}

Testing occurred over three sessions. First, a traditional assessment of 1RM for deadlift exercise was performed. Before attempting a $1 \mathrm{RM}$, subjects performed a specific deadlift warm-up, which consisted of eight repetitions

Figure 1 Execution of the deadlift exercise with hexagon barbell and measuring/positioning of the velocity-based training variables by PUSH device (arrow)

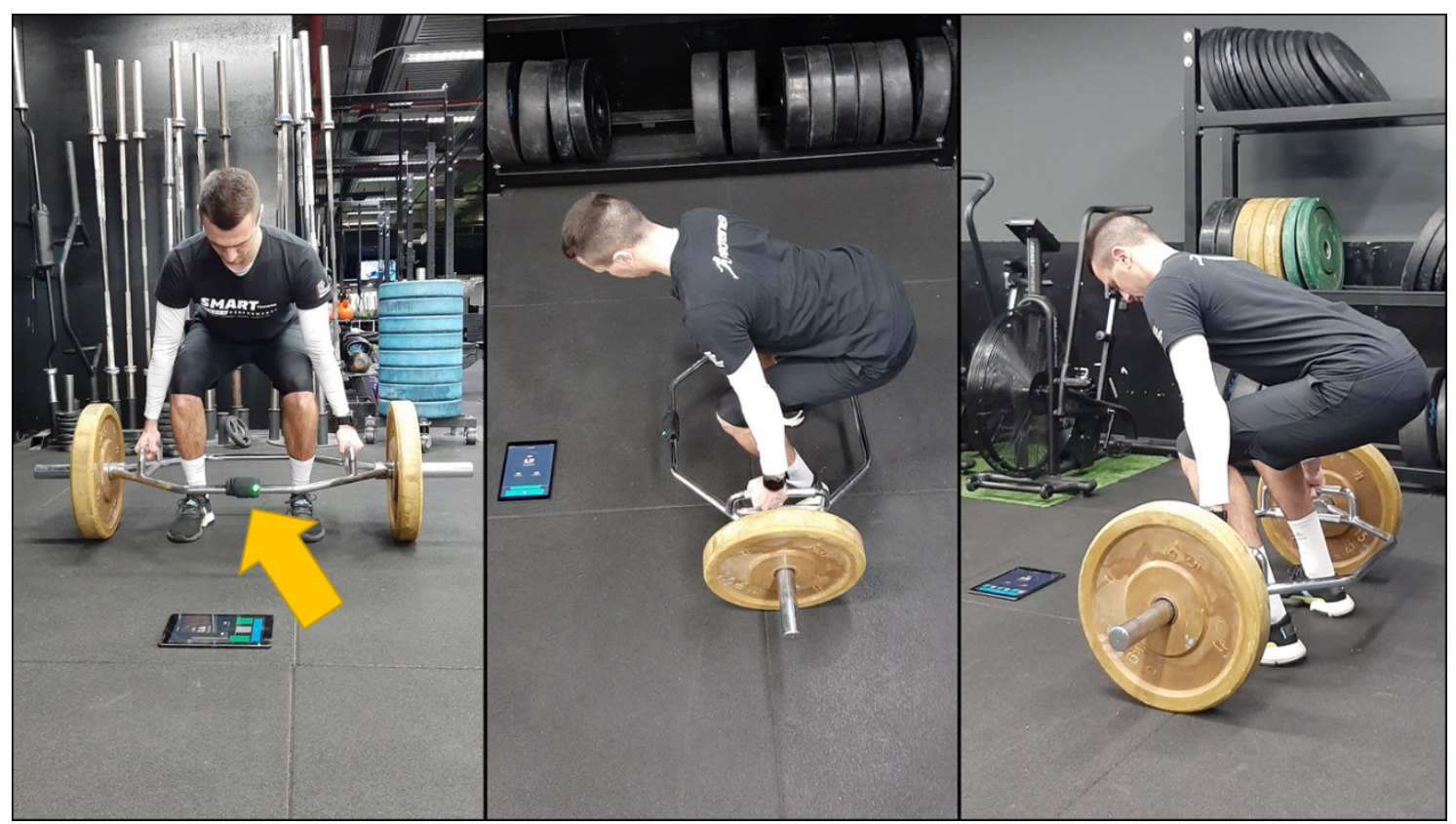


with an empty hexagonal barbell. After a two-minute rest, participants performed a further warm-up set of six repetitions with a relatively light load, then three repetitions with a heavier load, and finally a series of single repetitions with increasing loads. At higher loads, players performed one repetition and then rest for at least three minutes. If the weight was lifted with the proper form, the weight was increased by approximately $0.5-2.5 \mathrm{~kg}$, and the subject attempted repetition with a heavier load. The 1RM represented the maximal load that the participant was able to lift once (Faigenbaum et al., 2003). Traditional 1RM testing was performed in order to prescribe the load for the 1RM testing protocol using the PUSH Band.

Second, players participated in the familiarization session when all participants underwent tests for all variables in the specified order: anthropometric, SJ and BJ, and measuring VBT-variables during the deadlift incremental protocol.

Third, all players participated in an experimental session that was identical to the familiarization session in the same testing order. The traditional 1RM assessment, familiarization and experimental testing sessions were separated by seven days, and all players were tested between 9 and 11 am to avoid diurnal variation. Familiarization and experimental sessions followed the same testing order, which is explained in detail in the following text. The anthropometric variables were assessed before the warm-up. The warm-up lasted for 12 minutes and consisted of 5 minutes of cycling using the Keiser M3 (Keiser Corporation, CA, USA) stationary bicycle (70 RPM, 85 W), 2 minutes of simple drills on agility ladders, dynamic stretching, and mobility exercises including exercises for the hip adductors, hamstring muscles, gluteal muscles, and gastrocnemius muscle.

After warming up, the players underwent the SJ and BJ tests, and they performed three trials for each jump. Afterward, the incremental 1RM protocol with measuring VBT-variables was started. The load for each increment was prescribed based on the previously assessed 1RM (assessed during the first testing session).

First, the players performed $8-10$ repetitions of the deadlift with an empty hexagonal barbell, 5-6 repetitions at $20 \%$ of $1 \mathrm{RM}$, and $3-5$ repetitions at $30 \%$ of $1 \mathrm{RM}$. Next, the players started lifting loads at $45 \%$ (4 repetitions), 55\% (3 repetitions), 65\% (2 repetitions) of 1RM and then lifted loads at $75 \%, 85 \%$, and $95 \%$ of $1 \mathrm{RM}$ once (if the players were able to lift the given load). The players were instructed to lift the load as fast as possible while maintaining eccentric control. The players had 3-4 minutes of rest between each increment of load. During each repetition from $45 \%$ to $95 \%$ of $1 \mathrm{RM}$, a wearable accelerometer (PUSH Band 2.0) was attached to the hexagonal barbell to calculate the VBT-variables (velocity, force, power). The repetition with the highest velocity recorded during each load increment was used in further analysis.

\section{Statistics}

All data were log-transformed to reduce the non-uniformity of error, and normality was confirmed using the Kolmogorov-Smirnov test procedure. Homoscedasticity was checked by the Levene test. The statistical analyses were done on log-transformed data, but results in tables and figures are presented as true-value means and standard deviations.

In order to identify associations between variables Pearson's product-moment correlation coefficients were calculated between anthropometric and VBT-variables obtained at different loads, and jumping performances. Next, multiple regression analyses were calculated between predictors and criteria-jumping performances. Specifically, six multiple regression calculations were done for each observed jump (SJ and BJ). Irrespective of the univariate correlations, predictors in multiple regressions included body height, body mass, and body fat percentage, relative average power, and relative peak power obtained at each load since it was possible that some variables although not being univariately correlated to jumping performances will contribute to multivariate correlations to some extent. However, velocity and force variables were not included in the multiple regressions due to their high collinearity with power-variables (i.e., power variables are calculated on the basis of velocity, and force variables at each training load).

To identify the differences within each VBT-variable (for different loads), analysis of the variance for repeated measurements (ANOVA), and subsequent post-hoc Schefee test were calculated.

Statistica (Version 13; TIBCO, Palo Alto, CA, USA) was used for all calculations, and $p$-level of .05 was applied.

\section{Results}

Descriptive statistics and Pearson's correlation coefficients are presented in Table 1. Anthropometric variables were not significantly correlated with jumps, while most of the VBT-variables significantly correlate with both jumping performances. However, VBT-variables were more strongly correlated to $\mathrm{BJ}$ ( $31 \%$ to $70.5 \%$ of the common variance) than to SJ (27\% to $56 \%$ of the common variance; Table 1$)$.

Multivariate regressions between anthropometric indices and power-variables derived during VBT at different loads (predictors) and BJ (criterion) reached statistical significance for calculations including power-variables at $45 \% 1 \mathrm{RM}$ (74\% of the explained variance), 55\%1RM (69\% of the explained variance), 65\%1RM (64\% of the explained variance), $75 \% 1 \mathrm{RM}$ ( $67 \%$ of the common variance), and $85 \% 1 \mathrm{RM}$ (73\% of the common variance; Table 2$)$.

When multiple regressions between anthropometric indices and power-predictors derived at different loads of VBT were calculated for SJ as criterion variable, significant multivariate associations were found only for 55\%1RM (65\% of the explained variance; Table 3). Consequently, we may say that VBT-variables explained BJ-variance to a greater extent than SJ-variance.

Mean values and ANOVA differences within each VBTvariable evidenced at various training loads are presented in Figure 2. Statistically significant ANOVA effects were evidenced for the AF (with significant post-hoc differences when $45 \mathrm{AF}$ was compared to $85 \mathrm{AF}$ and $95 \mathrm{AF}$, and when $55 \mathrm{AF}$ was compared to $95 \mathrm{AF}$ ), for the AP and for the PP (significant post-hoc differences when performances at $45 \% 1 \mathrm{RM}, 55 \% 1 \mathrm{RM}$ and 65\%1RM were compared 
Table 1 Descriptive statistics and Pearson's moment correlation coefficients between studied variables

\begin{tabular}{|c|c|c|c|c|}
\hline \multirow[b]{2}{*}{ Variable } & \multirow[b]{2}{*}{$M$} & \multirow[b]{2}{*}{$S D$} & \multicolumn{2}{|c|}{$r$} \\
\hline & & & Sargent jump & Broad jump \\
\hline Deadlift 1RM (kg) & 103.13 & 17.97 & $.57 *$ & .46 \\
\hline $\mathrm{BH}(\mathrm{cm})$ & 181.25 & 5.99 & .45 & .42 \\
\hline $\mathrm{BM}(\mathrm{kg})$ & 67.12 & 7.14 & .38 & .35 \\
\hline$\% \mathrm{BF}(\% \mathrm{BM})$ & 14.61 & 2.27 & .10 & -.48 \\
\hline $45 \mathrm{AF}(\mathrm{N} / \mathrm{kg})$ & 24.60 & 2.50 & -.10 & .34 \\
\hline $45 \mathrm{PF}(\mathrm{N} / \mathrm{kg})$ & 28.57 & 2.99 & .27 & $.62^{*}$ \\
\hline 45AP (W/kg) & 15.97 & 3.77 & $.55^{*}$ & $.81^{* * *}$ \\
\hline $45 \mathrm{PP}(\mathrm{W} / \mathrm{kg})$ & 34.91 & 6.44 & $.52^{*}$ & $.84 * * *$ \\
\hline $45 \mathrm{AV}(\mathrm{m} / \mathrm{s})$ & 1.15 & 0.22 & $.75^{* * *}$ & $.73^{* * *}$ \\
\hline $45 \mathrm{PV}(\mathrm{m} / \mathrm{s})$ & 1.98 & 0.30 & $.73 * * *$ & $.76 * * *$ \\
\hline $55 \mathrm{AF}(\mathrm{N} / \mathrm{kg})$ & 25.09 & 2.39 & -.20 & .26 \\
\hline $55 \mathrm{PF}(\mathrm{N} / \mathrm{kg})$ & 29.53 & 3.99 & .11 & $.56^{*}$ \\
\hline $55 \mathrm{AP}(\mathrm{W} / \mathrm{kg})$ & 15.99 & 3.81 & $.61^{*}$ & $.73^{* * *}$ \\
\hline $55 \mathrm{PP}(\mathrm{W} / \mathrm{kg})$ & 35.00 & 7.53 & $.63 * *$ & $.64 * *$ \\
\hline $55 \mathrm{AV}(\mathrm{m} / \mathrm{s})$ & 1.05 & 0.21 & $.59 *$ & $.55^{*}$ \\
\hline $55 \mathrm{PV}(\mathrm{m} / \mathrm{s})$ & 1.82 & 0.34 & $.61^{*}$ & .48 \\
\hline $65 \mathrm{AF}(\mathrm{N} / \mathrm{kg})$ & 25.65 & 2.17 & .39 & .42 \\
\hline $65 \mathrm{PF}(\mathrm{N} / \mathrm{kg})$ & 30.00 & 4.65 & $.60^{*}$ & $.70^{* *}$ \\
\hline $65 \mathrm{AP}(\mathrm{W} / \mathrm{kg})$ & 15.44 & 3.99 & $.56^{*}$ & $.74^{* * *}$ \\
\hline $65 \mathrm{PP}(\mathrm{W} / \mathrm{kg})$ & 34.42 & 6.74 & $.56^{*}$ & $.71^{* *}$ \\
\hline $65 \mathrm{AV}(\mathrm{m} / \mathrm{s})$ & 0.98 & 0.21 & .40 & $.69 * *$ \\
\hline $65 \mathrm{PV}(\mathrm{m} / \mathrm{s})$ & 1.73 & 0.31 & .32 & $.56^{*}$ \\
\hline $75 \mathrm{AF}(\mathrm{N} / \mathrm{kg})$ & 25.80 & 2.14 & .22 & .06 \\
\hline $75 \mathrm{PF}(\mathrm{N} / \mathrm{kg})$ & 30.33 & 3.46 & .31 & .19 \\
\hline $75 \mathrm{AP}(\mathrm{W} / \mathrm{kg})$ & 13.46 & 4.21 & $.58^{*}$ & $.62 *$ \\
\hline $75 \mathrm{PP}(\mathrm{W} / \mathrm{kg})$ & 31.47 & 10.21 & $.64^{*}$ & $.73^{* * *}$ \\
\hline $75 \mathrm{AV}(\mathrm{m} / \mathrm{s})$ & 0.81 & 0.23 & .37 & .48 \\
\hline $75 \mathrm{PV}(\mathrm{m} / \mathrm{s})$ & 1.51 & 0.40 & .45 & $.63 * *$ \\
\hline $85 \mathrm{AF}(\mathrm{N} / \mathrm{kg})$ & 27.07 & 2.38 & .03 & .20 \\
\hline $85 \mathrm{PF}(\mathrm{N} / \mathrm{kg})$ & 30.97 & 3.88 & .17 & .40 \\
\hline $85 \mathrm{AP}(\mathrm{W} / \mathrm{kg})$ & 12.09 & 4.86 & $.55^{*}$ & $.71 * *$ \\
\hline $85 \mathrm{PP}(\mathrm{W} / \mathrm{kg})$ & 27.09 & 10.35 & $.54^{*}$ & $.71^{* *}$ \\
\hline $85 \mathrm{AV}(\mathrm{m} / \mathrm{s})$ & 0.66 & 0.23 & .50 & $.68^{* *}$ \\
\hline $85 \mathrm{PV}(\mathrm{m} / \mathrm{s})$ & 1.22 & 0.38 & .49 & $.67^{* *}$ \\
\hline $95 \mathrm{AF}(\mathrm{N} / \mathrm{kg})$ & 28.25 & 1.77 & .00 & .06 \\
\hline $95 \mathrm{PF}(\mathrm{N} / \mathrm{kg})$ & 30.98 & 2.47 & .38 & .57 \\
\hline 95AP (W/kg) & 11.32 & 3.72 & .47 & $.79 * *$ \\
\hline $95 \mathrm{PP}(\mathrm{W} / \mathrm{kg})$ & 24.98 & 10.54 & .54 & $.79 * *$ \\
\hline $95 \mathrm{AV}(\mathrm{m} / \mathrm{s})$ & 0.60 & 0.22 & .35 & $.66^{*}$ \\
\hline $95 \mathrm{PV}(\mathrm{m} / \mathrm{s})$ & 1.10 & 0.43 & .41 & $.69 *$ \\
\hline Sargent jump (cm) & 47.56 & 5.74 & - & $.64 * *$ \\
\hline Broad jump $(\mathrm{cm})$ & 226.80 & 18.17 & $.64^{* *}$ & - \\
\hline
\end{tabular}

Note. $1 \mathrm{RM}=$ one-repetition maximum; $\mathrm{BH}=$ body height; $\mathrm{BM}=$ body mass; $\% \mathrm{BF}=$ body fat percentage; $\mathrm{AF}=$ average force; $\mathrm{PF}=$ peak force; $\mathrm{AP}=$ average power; $\mathrm{PP}=$ peak power; $\mathrm{AV}=$ average velocity; $\mathrm{PV}=$ peak velocity at $45,55,65$, 75,85 , and $95 \%$ of 1 RM. $^{*} p<.05,{ }^{* *} p<.01,{ }^{* *} p<.001$.

to performance at 95\%1RM), for the AV and for the PV (significant post-hoc differences when performances at $45 \% 1 \mathrm{RM}$ were compared with performances at $75 \% 1 \mathrm{RM}$, $85 \% 1 \mathrm{RM}$ and $95 \% 1 \mathrm{RM}$, when performances at 55\%1RM and 65\%1RM were compared with performances at $85 \% 1 \mathrm{RM}$ and $95 \% 1 \mathrm{RM}$, and when performance at $75 \% 1 \mathrm{RM}$ was compared with performance at 95\%1RM).

\section{Discussion}

This study aimed to investigate the correlations between VBT-variables and jumping performance in young athletes. We found stronger correlations between the $\mathrm{BJ}$ and VBT-variables than between the SJ and VBT-variables. Also, VBT-variables obtained while performing the deadlift with lighter loads stronger correlated with jumping performances than the VBT-variables obtained at higher loads did. Therefore, our initial study hypothesis was supported.

\section{Correlations between VBT-variables and different jumps}

The VBT-variables derived during the execution of the deadlift were more strongly correlated with the BJ than with the SJ. This finding is explainable considering the similarity between deadlift and BJ performance. Specifically, it is well known that muscle activation differs between the SJ and BJ. Early experimental studies have demonstrated differences between horizontal and vertical jumps in the contributions of different joints $(45.9 \%, 3.9 \%$, and $50.2 \%$ vs. $40 \%, 24.2 \%$, and $35.8 \%$ for the hip joint, knee joint, and ankle joint in the horizontal and vertical jumps, respectively; Robertson \& Fleming, 1987). Moreover, the range of motion and angular velocity of the hip joint are larger during the horizontal jump, while the range of motion and angular velocity of the knee joint are larger during the vertical jump (Eckert, 1968). Finally, it has been shown that the magnitude of hip flexion, total range of motion of the hip joint, and force generated by the hip muscles are larger in the horizontal jump than in the vertical jump (Nagano et al., 2007). The deadlift is a multijoint exercise where the largest muscular moments are produced mostly at the hip joint, followed by the lumbar spine, ankle, and knee (Brown \& Abani, 1985). Therefore, it is clear that the deadlift is biomechanically more similar to the $\mathrm{BJ}$ than to the SJ, which probably resulted in the stronger correlations between the BJ and VBT-variables in our study.

We must note that the similarity between the BJ and VBT in our experiment was additionally aggravated by the fact that the participants used a hexagonal barbell for the deadlift exercise. Deadlifts with this training tool mimic jumping to a greater extent than deadlifts performed with the classic Olympic barbell. In brief, performing deadlifts with a hexagonal barbell is more similar to jumping because the body position is more upright, and it leads to larger knee flexion and increased quadriceps muscle activation. Moreover, the angle of the hip joint and hip muscle activation are very pronounced when deadlifts are performed with a hexagonal barbell (Swinton et al., 2011). As during the horizontal jump, movement in the hip joint is more pronounced, this could be the reason for stronger correlations between deadlift performed with hexagonal barbell and horizontal jump. In general, the stress on the lumbar spine is reduced, and it is possible to lift heavier loads and to generate higher levels of velocity, force, and power with the hexagonal barbell than with the traditional straight Olympic barbell (Camara et al., 2016; Swinton et al., 2011). These factors almost certainly contributed to the 
Table 2 Multivariate associations between anthropometric variables and power variables derived at different loads (45\%-95\% of 1RM), and broad jump performance

\begin{tabular}{|c|c|c|c|c|c|c|c|c|c|c|c|c|}
\hline \multirow[b]{2}{*}{ Variable } & \multicolumn{2}{|c|}{$45 \%$ of $1 \mathrm{RM}$} & \multicolumn{2}{|c|}{$55 \%$ of $1 \mathrm{RM}$} & \multicolumn{2}{|c|}{$65 \%$ of $1 \mathrm{RM}$} & \multicolumn{2}{|c|}{$75 \%$ of $1 \mathrm{RM}$} & \multicolumn{2}{|c|}{$85 \%$ of $1 \mathrm{RM}$} & \multicolumn{2}{|c|}{$95 \%$ of $1 \mathrm{RM}$} \\
\hline & $\beta$ & $b$ & $\beta$ & $b$ & $\beta$ & $b$ & $\beta$ & $b$ & $\beta$ & $b$ & $\beta$ & $b$ \\
\hline $\mathrm{BH}$ & -0.02 & -0.06 & 0.07 & 0.20 & -0.09 & -0.28 & 0.00 & 1.20 & 0.14 & 0.42 & 0.18 & 0.55 \\
\hline BM & 0.12 & 0.30 & 0.18 & 0.45 & 0.18 & 0.46 & 0.14 & 1.03 & 0.17 & 0.44 & -0.27 & -0.70 \\
\hline$\% \mathrm{BF}$ & -0.17 & -1.37 & -0.32 & -2.52 & -0.30 & -2.41 & -0.28 & 1.73 & -0.35 & -2.79 & -0.22 & -1.78 \\
\hline AP & 0.08 & 0.37 & 0.49 & 2.35 & 0.61 & 2.79 & -0.37 & 3.00 & 0.83 & 3.12 & 0.25 & 1.26 \\
\hline PP & 0.67 & 1.90 & 0.15 & 0.37 & 0.03 & 0.07 & 0.98 & 1.22 & -0.20 & -0.35 & 0.55 & 0.97 \\
\hline$R$ & .86 & & .83 & & .80 & & .81 & & .86 & & .85 & \\
\hline$R^{2}$ & .74 & & .69 & & .64 & & .67 & & .73 & & .73 & \\
\hline$p$ & .01 & & .02 & & .04 & & .03 & & .02 & & .25 & \\
\hline
\end{tabular}

Note. $1 \mathrm{RM}=$ one-repetition maximum; $\beta=$ standardized regression coefficient; $b=$ nonstandardized regression coefficient; $\mathrm{BH}=$ body height; $\mathrm{BM}=$ body mass; $\% \mathrm{BF}=$ body fat percentage; $\mathrm{AP}=$ average power; $\mathrm{PP}=$ peak power; $R=$ multiple regression; $R^{2}=$ coefficient of determination.

Table 3 Multivariate associations between anthropometric variables and power variables derived at different loads (45\%-95\% of 1RM), and Sargent jump performance

\begin{tabular}{|c|c|c|c|c|c|c|c|c|c|c|c|c|}
\hline \multirow[b]{2}{*}{ Variable } & \multicolumn{2}{|c|}{$45 \%$ of $1 \mathrm{RM}$} & \multicolumn{2}{|c|}{$55 \%$ of $1 \mathrm{RM}$} & \multicolumn{2}{|c|}{$65 \%$ of $1 \mathrm{RM}$} & \multicolumn{2}{|c|}{$75 \%$ of $1 \mathrm{RM}$} & \multicolumn{2}{|c|}{$85 \%$ of $1 \mathrm{RM}$} & \multicolumn{2}{|c|}{$95 \%$ of $1 \mathrm{RM}$} \\
\hline & $\beta$ & $b$ & $\beta$ & $b$ & $\beta$ & $b$ & $\beta$ & $b$ & $\beta$ & $b$ & $\beta$ & $b$ \\
\hline $\mathrm{BH}$ & 0.53 & 0.51 & 0.57 & 0.55 & 0.41 & 0.39 & 0.46 & 0.44 & 0.63 & 0.60 & 0.65 & 0.62 \\
\hline BM & -0.22 & -0.18 & -0.13 & -0.10 & -0.12 & -0.10 & -0.11 & -0.09 & -0.13 & -0.10 & -0.43 & -0.35 \\
\hline$\% \mathrm{BF}$ & 0.47 & 1.19 & 0.36 & 0.92 & 0.45 & 1.15 & 0.44 & 1.11 & 0.35 & 0.88 & 0.41 & 1.03 \\
\hline AP & 0.36 & 0.54 & 0.28 & 0.42 & 0.14 & 0.21 & -0.56 & -0.76 & 0.56 & 0.66 & 0.05 & 0.08 \\
\hline PP & 0.24 & 0.21 & 0.37 & 0.28 & 0.47 & 0.40 & 1.13 & 0.64 & -0.01 & -0.01 & 0.51 & 0.28 \\
\hline$R$ & .72 & & .81 & & .73 & & .78 & & .76 & & .50 & \\
\hline$R^{2}$ & .52 & & .65 & & .53 & & .61 & & .57 & & .25 & \\
\hline$p$ & .14 & & .04 & & .13 & & .06 & & .12 & & .64 & \\
\hline
\end{tabular}

Note. $1 \mathrm{RM}=$ one-repetition maximum; $\beta=$ standardized regression coefficient; $b=$ nonstandardized regression coefficient; $\mathrm{BH}=$ body height; $\mathrm{BM}=$ body mass; $\% \mathrm{BF}=$ body fat percentage; $\mathrm{AP}=$ average power; $\mathrm{PP}=$ peak power; $R=$ multiple regression; $R^{2}=$ coefficient of determination.

association between the VBT-variables of the deadlift and horizontal jumping performance.

Correlations between VBT-variables obtained at different loads and jumping performances

The second significant finding of this study is that the VBTvariables derived while performing deadlifts with lighter loads (45\%-65\% of 1RM) were better determinants of jumping performance than were the VBT-variables derived at higher loads. Although we could not find a study where correlations between VBT-variables at different loads and jumping performance were directly examined, our results are consistent with those of studies where the authors directly examined power-output using the different loads. Specifically, Baker et al. (2001) noted that moderate loads in the range from 48 to $63 \%$ of $1 \mathrm{RM}$ evoked the highest power output during the concentric part of the jump squat exercise.

The strong correlations between jumping performance and VBT-variables obtained with lighter loads are probably related to the fact that loads higher than approximately $65 \%$ of $1 \mathrm{RM}$ result in a proportional decrease in velocity, which leads to decreased power output. On the other hand, lighter loads $(<45 \%$ of $1 \mathrm{RM})$ result in higher velocities, but the lighter mass leads to decreased power output (Baker et al., 2001). Similarly, velocity values decreased proportionally with increasing loads in our study. Specifically, in our study, the highest velocity was recorded when a deadlift at $45 \%$ of $1 \mathrm{RM}$ was performed, while the lowest velocity was recorded at $95 \%$ of $1 \mathrm{RM}$. Moreover, the power values were the highest in the range from $45 \%$ to $65 \%$ of $1 \mathrm{RM}$ and started decreasing thereafter (with increasing load).

Another possible explanation for the strong correlations between the VBT-variables at lower loads and jumping performance is that this research included youth players. Although the participants were involved in strength training for a substantial period, they were not considered highly experienced in training with external loads. Therefore, there is a possibility that their motor proficiency at the highest loads ( $85 \%$ and $95 \%$ of $1 \mathrm{RM}$ ) was not at the appropriate technical level, and consequently, it is possible that the 1RM assessed in this study does not represent the real condition for each player. Supportively, Ritti-Dias et al. (2011) suggested that people who do not have much experience in resistance training are prone to displaying changes (e.g., improvements) in maximal strength after repeated testing due to familiarization rather than changes in strength capacity alone. Therefore, athletes less experienced in performing resistance training might not show precise and accurate maximum-strength values, which could have influenced our results to some extent. 
Figure 2 Descriptive statistics (means $\pm 95 \%$ confidence intervals) and results of analysis of the variance for repeated measurements of variables derived at velocity-based training with different loads
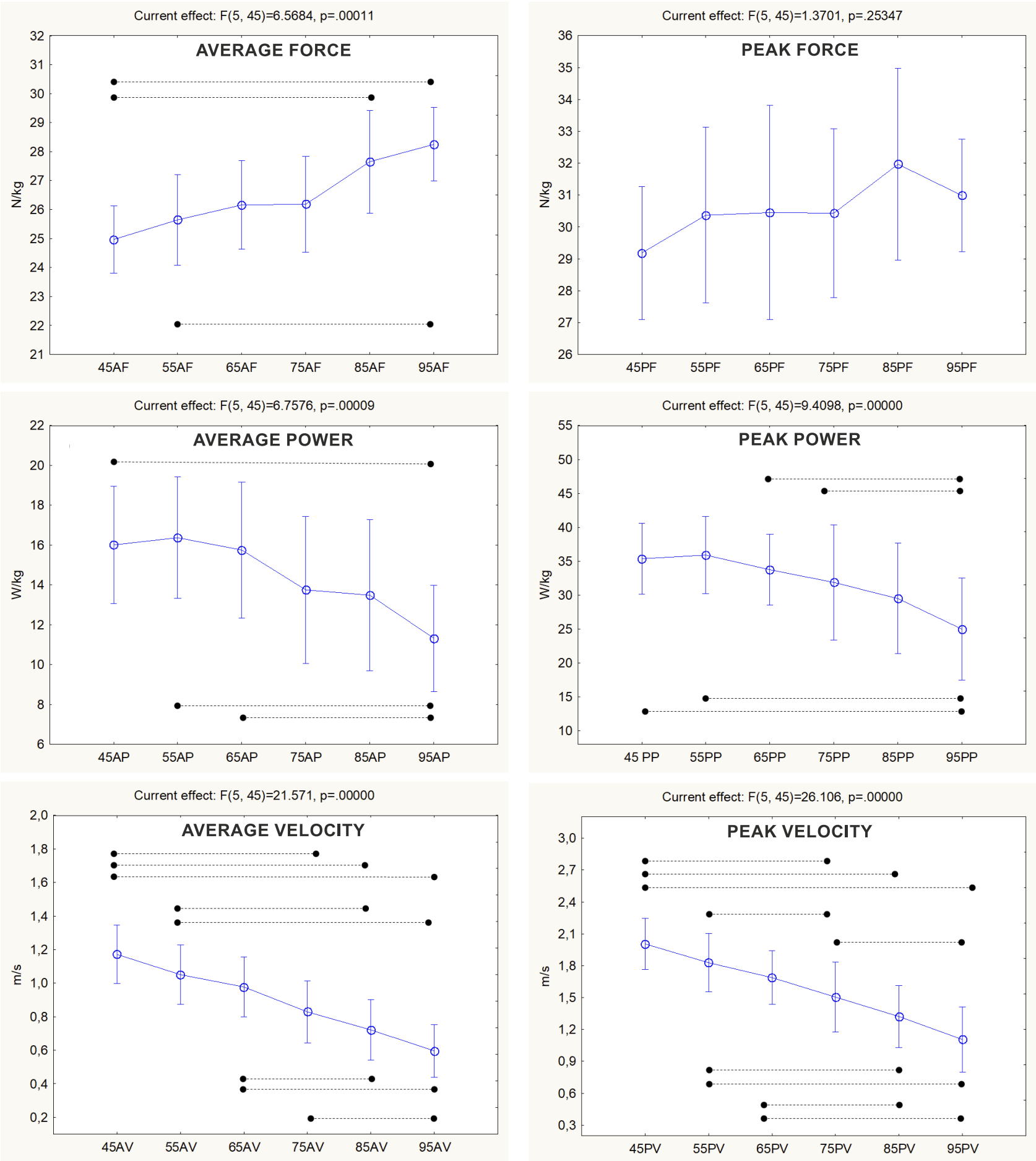

Note. $\mathrm{AF}=$ average force relative to body mass of the player; $\mathrm{PF}=$ peak force relative to body mass of the player; $\mathrm{AP}=$ average power relative to body mass of the player; $\mathrm{PP}=$ peak power relative to body mass of the player; $\mathrm{AV}=$ average velocity; $\mathrm{PV}=$ peak velocity at $45,55,65,75,85,95 \%$ of one-repetition maximum. Dotted connectors present significant post-hoc differences between measurements.

\section{Limitations and strengths}

The main limitation is that the study had a cross-sectional design. Therefore, longitudinal analyses are needed to investigate the effects of VBT deadlift performance at different loads on performance in various types of jumps. Furthermore, this study included young soccer players who had a relatively short period of experience in resistance training. Therefore, the results can be generalized to only athletes with similar levels of experience in weight training. Another limitation of the study is associated with the execution of the jumps. Precisely, both jumps were performed with arm swing, which could influence (i.e., decrease) the correlation 
between VBT-variables derived during the deadlift and observed jumps. Also, the possible contribution of specific coordination necessary for jumps cannot be ignored. However, tested participants were relatively experienced athletes (minimally eight years of soccer training) and, therefore, possess an adequate level of specific coordination necessary for jumping performance. Also, we aimed to evaluate "reallife" jumping performances which are regularly performed with arm swing.

This is one of the first studies where the relationship between performance in different jumps and VBT-variables derived at different loads were observed. Therefore, the results can contribute to our understanding of the relationship between the observed variables. More importantly, the results could be used for developing targeted interventions in which the optimal deadlift load that will improve (specific) jumping performance is prescribed.

\section{Conclusions}

Our results showed that VBT-variables recoded during exercises with light-to-moderate loads are more strongly related to jumping performance than are those recorded with high loads. However, it is important to mention that the results of our study do not suggest that heavier loads should not be used in training, but it was observed that light-to-moderate loads (45\%-65\% of $1 \mathrm{RM}$ ) seem more likely to maximize power output and were consequently more strongly related to jumping performance.

Considering that our study included youth soccer players who had limited experience in resistance training with high loads, it is important to emphasize that the results of this study should not be generalized to all athletes. Specifically, there is a possibility that among older players and those with more experience in resistance training, the results are not the same. Therefore, to verify these results, future studies in different athletes and populations are warranted.

\section{Acknowledgments}

The authors are particularly grateful to all players who voluntarily participated in this study.

\section{Conflict of interest}

The authors report no conflict of interest.

\section{References}

Baker, D., Nance, S., \& Moore, M. (2001). The load that maximizes the average mechanical power output during jump squats in power-trained athletes. Journal of Strength and Conditioning Research, 15(1), 92-97. https://journals.lww. $\mathrm{com} / \mathrm{nsca-jscr} / \mathrm{abstract} / 2001 / 02000 /$ the load that maximizes the average mechanical.16.aspx

Balsalobre-Fernández, C., Kuzdub, M., Poveda-Ortiz, P., \& Campo-Vecino, J. D. (2016). Validity and reliability of the PUSH wearable device to measure movement velocity during the back squat exercise. Journal of Strength and Conditioning Research, 30(7), 1968-1974. https://doi.org/10.1519/isc.0000000000001284

Banyard, H. G., Tufano, J. J., Delgado, J., Thompson, S. W., \& Nosaka, K. (2019). Comparison of the effects of velocity-based training methods and traditional 1RM-percent-based training prescription on acute kinetic and kinematic variables. International Journal of Sports Physiology and Performance, 14(2), 246-255. https://doi.org/10.1123/ijspp.2018-0147
Bendic, V., Gilic, B., \& Sekulic, D. (2021). Associations between vertical and horizontal jumps with power values during deadlift exercise among youth soccer (football) players. Asian Exercise and Sport Science Journal, 5(1), 55-60. https://doi.org/10.30472/aesj.v5i1.222

Brown, E. W., \& Abani, K. (1985). Kinematics and kinetics of the dead lift in adolescent power lifters. Medicine \& Science in Sports \& Exercise, 17(5), 554-566. https://journals.Iww.com/acsm-msse/Abstract/1985/10000/Kinematics and_kinetics of the dead lift in.8.aspx

Camara, K. D., Coburn, J. W., Dunnick, D. D., Brown, L. E., Galpin, A. J., \& Costa, P. B. (2016). An examination of muscle activation and power characteristics while performing the deadlift exercise with straight and hexagonal barbells. Journal of Strength and Conditioning Research, 30(5), 1183-1188. https://doi. org/10.1519/isc.0000000000001352

Chéry, C., \& Ruf, L. (2019). Reliability of the load-velocity relationship and validity of the PUSH to measure velocity in the deadlift. Journal of Strength and Conditioning Research, 33(9), 2370-2380. https://doi.org/10.1519/isc.0000000000002663 de Salles, P. G., Vasconcellos, F. V., de Salles, G. F., Fonseca, R. T., \& Dantas, E. H. (2012). Validity and reproducibility of the Sargent Jump Test in the assessment of explosive strength in soccer players. Journal of Human Kinetics, 33, 115-121. https://doi.org/10.2478/v10078-012-0050-4

Dolezal, S. M., Frese, D. L., \& Llewellyn, T. L. (2016). The effects of eccentric, velocity-based training on strength and power in collegiate athletes. International Journal of Exercise Science, 9(5), 657-666. https://digitalcommons.wku.edu/ ijes/vol9/iss5/12/

Dorrell, H. F., Smith, M. F., \& Gee, T. I. (2020). Comparison of velocity-based and traditional percentage-based loading methods on maximal strength and power adaptations. Journal of Strength and Conditioning Research, 34(1), 46-53. https://doi.org/10.1519/isc.0000000000003089

Eckert, H. M. (1968). Angular velocity and range of motion in the vertical and standing broad jumps. Research Quarterly - American Association for Health, Physical Education and Recreation, 39(4), 937-942. https://doi.org/10.1080/1067 1188.1968.10613442

Faigenbaum, A. D., Milliken, L. A., \& Westcott, W. L. (2003). Maximal strength testing in healthy children. Journal of Strength and Conditioning Research, 17(1), 162-166. https://doi.org/10.1519/00124278-200302000-00025

González-Badillo, J. J., Pareja-Blanco, F., Rodríguez-Rosell, D., Abad-Herencia, J. L., Del Ojo-López, J. J., \& Sánchez-Medina, L. (2015). Effects of velocitybased resistance training on young soccer players of different ages. Journal of Strength and Conditioning Research, 29(5), 1329-1338. https://doi.org/10.1519/ jsc. 0000000000000764

Helms, E. R., Byrnes, R. K., Cooke, D. M., Haischer, M. H., Carzoli, J. P., Johnson, T. K., Cross, R. M., Cronin, J. B., Storey, A. G., \& Zourdos, M. C. (2018). RPE vs. percentage $1 \mathrm{RM}$ loading in periodized programs matched for sets and repetitions. Frontiers in Physiology, 9, Article 247. https://doi.org/10.3389/fphys.2018.00247

Hughes, L. J., Banyard, H. G., Dempsey, A. R., Peiffer, J. J., \& Scott, B. R. (2019). Using load-velocity relationships to quantify training-induced fatigue. Journal of Strength and Conditioning Research, 33(3), 762-773. https://doi.org/10.1519/ jsc. 0000000000003007

Jovanovic, M., \& Flanagan, E. P. (2014). Researched applications of velocity based strength training. Journal of Australian Strength and Conditioning, 22(2), 58-69.

Malyszek, K. K., Harmon, R. A., Dunnick, D. D., Costa, P. B., Coburn, J. W., \& Brown, L. E. (2017). Comparison of Olympic and hexagonal barbells with midthigh pull, deadlift, and countermovement jump. Journal of Strength and Conditioning Research, 31(1), 140-145. https://doi.org/10.1519/jsc.0000000000001485

Mann, J. B., Ivey, P. A., \& Sayers, S. P. (2015). Velocity-based training in football. Strength and Conditioning Journal, 37(6), 52-57. https://doi.org/10.1519/ ssc.00000000000000177

Nagano, A., Komura, T., \& Fukashiro, S. (2007). Optimal coordination of maximaleffort horizontal and vertical jump motions - A computer simulation study. BioMedical Engineering OnLine, 6, Article 20. https://doi.org/10.1186/1475-925X-6-20 Orange, S. T., Metcalfe, J. W., Robinson, A., Applegarth, M. J., \& Liefeith, A. (2020). Effects of in-season velocity-versus percentage-based training in academy rugby league players. International Journal of Sports Physiology and Performance, 15(4), 554-561. https://doi.org/10.1123/ijspp.2019-0058

Ortega, J. A. F., De los Reyes, Y. G., \& Peña, F. R. G. (2020). Effects of strength training based on velocity versus traditional training on muscle mass, neuromuscular activation, and indicators of maximal power and strength in girls soccer players. Apunts Sports Medicine, 55(206), 53-61. https://doi.org/10.1016/j. apunsm.2020.03.002

Pehar, M., Sekulic, D., Sisic, N., Spasic, M., Uljevic, O., Krolo, A., Milanovic, Z., \& Sattler, T. (2017). Evaluation of different jumping tests in defining positionspecific and performance-level differences in high level basketball players. Biology of Sport, 34(3), 263-272. https://doi.org/10.5114/biolsport.2017.67122

Picerno, P., lannetta, D., Comotto, S., Donati, M., Pecoraro, F., Zok, M., Tollis, G., Figura, M., Varalda, C., Di Muzio, D., Patrizio, F., \& Piacentini, M. F. (2016). 1RM prediction: A novel methodology based on the force-velocity and load-velocity relationships. European Journal of Applied Physiology, 116(10), 2035-2043. https://doi.org/10.1007/s00421-016-3457-0

Randell, A. D., Cronin, J. B., Keogh, J. W., Gill, N. D., \& Pedersen, M. C. (2011). Effect of instantaneous performance feedback during 6 weeks of velocitybased resistance training on sport-specific performance tests. Journal of Strength and Conditioning Research, 25(1), 87-93. https://doi.org/10.1519/ ISC.0b013e3181fee634 
Ritti-Dias, R. M., Avelar, A., Salvador, E. P., \& Cyrino, E. S. (2011). Influence of previous experience on resistance training on reliability of one-repetition maximum test. Journal of Strength and Conditioning Research, 25(5), 1418-1422. https://doi.org/10.1519/JSC.0b013e3181d67c4b

Robertson, D. G., \& Fleming, D. (1987). Kinetics of standing broad and vertical jumping. Canadian Journal of Sport Sciences, 12(1), 19-23.

Schoenfeld, B. J., Ogborn, D., \& Krieger, J. W. (2016). Effects of resistance training frequency on measures of muscle hypertrophy: A systematic review and meta-analysis. Sports Medicine, 46(11), 1689-1697. https://doi.org/10.1007/ s40279-016-0543-8

Swinton, P. A., Stewart, A., Agouris, I., Keogh, J. W., \& Lloyd, R. (2011). A biomechanical analysis of straight and hexagonal barbell deadlifts using submaximal loads. Journal of Strength and Conditioning Research, 25(7), 2000-2009. https://doi.org/10.1519/JSC.0b013e3181e73f87

van den Tillaar, R., \& Ball, N. (2019). Validity and reliability of kinematics measured with PUSH band vs. linear encoder in bench press and push-ups. Sports, 7(9), https://doi.org/10.3390/sports7090207 